Description of the problem Footwear selected to be used in the mine work is normally based on the risk assessment because some workers do occasionally work that includes welding the footwear are selected on those based on those criteria's. Most commonly the footwear soles are selected so that they are resistant to hot contact (HRO) according the PPE-footwear standard. This leads to the selection of footwear which sole are made from dense materials like nitrile rubber. Using of those dense polymers like can be problematic in the arctic area because the slip resistance of the footwear soles made from dense materials is found to be poorer than footwear made from softer soling materials. The cold climate can increase the hardness of the soling material which makes footwear even more slippery.

Slip resistance, i.e. dynamic coefficient of friction (DCOF) of the footwear was measured in laboratory by using FIOH's own laboratory device slip simulator. Measurements were carried out according the parameters defined in the standard EN ISO 13287: 2012 Personal protective equipment-Footwear-Test method for slip resistance. The standard method was modified only by using smooth ice surface as a test surface. Ten consecutive measurements were done by each footwear model. The mean value and standard deviation was calculated. In addition the footwear was pre-conditioned in the climatic chamber at the temperature of $20 \mathrm{C}^{\circ}$ for four hours in order to see how the hardening of the sole materials affects to the slip resistance. Hardness of the sole was measured by using hardness tester with Shore (A) hardness scale.

Four different footwear were selected and collected form the mines participating in the project. Three of those footwear were safety footwear according EN ISO 20345: 2011 they were halfknee height leather footwear designed for winter conditions. One sample was rubber boot from Russia and it was not classified as safety footwear according to EN or ISO standards. Additionally six different safety footwear for winter conditions were selected in order to compare level of performance the slip resistance of footwear used in mines to the winter footwear available in the market.

Results Samples collected from the four mines got almost equal DCOF levels than the winter safety footwear selection from market when they were measured without pre-conditioning, but the DCOF values were 39\% lower compared to winter safety footwear selection when the samples were pre continued in climatic chamber. Hardness of the footwear sole increased 26\% in average because of the pre-conditioning in cold.

Conclusions Footwear currently used in the four mines have reasonable good slip resistance compared to footwear's available in the market, but their fiction level is strongly dependent of the temperature. For safety reasons, it would be advisable to use footwear sole materials which are not so much affected by the temperature changes especially in the artic area mines.

\section{PHYSICAL STRAIN OF MAST AND POLE WORK IN ARCTIC CONDITIONS}

${ }^{1}$ Juha Oksa, 'Sanna Hosio, ${ }^{1}$ Hannu Rintamäki, ' Sirkka Rissanen, ${ }^{2}$ Panu Oksa. ${ }^{1}$ Finnish Institute of Occupational Health, Oulu, Finland; ' ${ }^{2}$ Finnish Institute of Occupational Health, Tampere, Finland

\subsection{6/injuryprev-2016-042156.635}

Background Since physical strain during mast and pole work is not known this study evaluated the level of muscular, cardiorespiratory and thermal strain of mast and pole workers with special emphasis on arctic conditions, winter.
Methods Fourteen voluntary mast and pole workers participated. We measured their muscular strain using electromyography, expressed as percentage in relation to maximal EMG activity (\% MEMG). We estimated $\mathrm{VO}_{2}$ from $\mathrm{HR}$ measured during work (using individual $\mathrm{VO}_{2}$ - $\mathrm{HR}$ relationship) and expressed it as $\%$ $\mathrm{VO}_{2}$ max. To quantify thermal strain skin and deep body temperatures were measured using temperature sensors and telemetric pill and receiver.

Results We found the highest average muscular strain in the wrist flexor $(24 \pm 2 \%$ MEMG $)$ and extensor $(21 \pm 1 \%$ MEMG $)$ muscles, exceeding the recommendation of 14\% MEMG. Average cardiorespiratory strain was $48 \pm 3 \% \mathrm{VO}_{2}$ max. Nearly half $(40 \%)$ of the subjects exceeded the recommended $50 \% \mathrm{VO}_{2} \max$. Winter condition increased both muscular and cardiovascular strain on average by 4 and 2\%, respectively. Deep body temperature varied between 36.8 and $38.0^{\circ} \mathrm{C}$ and mean skin temperature between 28.6 and $33.4{ }^{\circ} \mathrm{C}$. Cooling was most pronounced in extremities during winter. Lowest single temperatures in middle finger, hand and big toe varied between 6.4 and 18.5, 9.4 and 24.9 and 15.4 and $24.6^{\circ} \mathrm{C}$, respectively.

Conclusions This field study showed that workers may be at risk for local and/or systemic muscular and cardiorespiratory overloading (the winter enhancing this effect) and thus for excessive fatigue, reduced work efficiency and increased risk for musculoskeletal symptoms. Generally, thermal strain remained at a tolerable level.

\section{STAY STANDING - WINTER SAFETY CAMPAIGN FOR PEDESTRIANS}

${ }^{1}$ Kaarina Tamminiemi, ${ }^{2}$ Tiina Tikkanen. ${ }^{1}$ SOSTE Finnish Federation for Social Affairs and Health, Finland; ${ }^{2}$ Finnish Red Cross, Finland

\subsection{6/injuryprev-2016-042156.636}

Background Stay Standing - Winter Safety Campaign for Pedestrians is part of the Implementation Project on Prevention of Home and Leisure Injuries. SOSTE Finnish Federation for Social Affairs and Health is the chairman of the campaign and Finnish Red Cross is coordinating the entire project. Partners in the campaign include various non-governmental, governmental and private organisations. Partners are working in the fields such as injury prevention, traffic safety and weather and climate conditions.

Description of the problem Each winter every four out of ten of the Finns (total population 5.2 million) are slipping due to icy weather conditions. Half of them get injured. Slipping causes plenty of minor injuries. Slipping also causes severe injuries such as fractures, dislocation of joints and head injuries, which may lead to long term disability. Each year more than 5000 people are hospitalised overnight during icy or snowy weather conditions. Slipping does not vary among the adult age group.

Results The Campaign promotes safety through informing the associated risks, which rise throughout the winter. The campaign also provides information on risk reduction. January is the most active month of the campaign. The target group is chosen each year. Also, different communication channels for the campaign are chosen each year: TV-spots, radio, digi screen, printed material and seminars.

Conclusions Conducting the campaign in collaboration with various governmental and non-governmental organisations has been very useful. By effective communication, the campaign has 